\title{
CARBONIZAÇÃO CONTÍNUA: PERFORMANCE DE UM ALTO-FORNO*
}

\author{
Rosiane Mary Rezende Faleiro ${ }^{1}$ \\ Cláudio Musso Velloso ${ }^{2}$ \\ Ronaldo Santos Sampaio ${ }^{3}$ \\ Flávio Roberto Silva de Azevedo ${ }^{4}$ \\ Fernando Lopes Latorre ${ }^{5}$
}

\section{Resumo}

O carvão vegetal, $\mathrm{CV}$, é o componente individual com maior impacto no custo final de uma usina integrada. Diante desta realidade, foi desenvolvido um processo de carbonização contínua, uma tecnologia moderna que a diferencia das carbonizações tradicionais, permitindo que o CV produzido tenha melhores características físicoquímicas. Sendo assim, o desafio agora é encontrar condições operacionais adequadas para produzir em larga escala a um custo competitivo. Com base nisso, desenvolveu-se este trabalho com o objetivo de avaliar o desempenho de um altoforno, em condições reais de operação e em escala industrial, utilizando dois tipos de carvões (CVs): CV Referência e CV produzido por carbonização contínua (Carboval). Os principais parâmetros operacionais foram monitorados. O CV Carboval apresentou melhores resultados para as análises químicas e granulométrica, melhor permeabilidade no alto-forno e menor consumo bruto, demonstrando sua viabilidade técnica.

Palavras-chave: Carvão vegetal; Carbonização contínua; Alto-forno; Desempenho.

\section{CONTINUOUS CARBONIZATION: BLAST FURNACE PERFORMANCE}

\begin{abstract}
Charcoal is the individual component that has the most impact on the final cost of an integrated plant. Given this reality, it was developed a continuous carbonization process, a modern technology that differs from traditional carbonization, allowed that the charcoal produced has better physicochemical characteristics. So the challenge now is to find appropriate operating conditions to produce in large-scale at a competitive cost. Based on this, it was developed this work in order to evaluate the performance of a blast furnace in actual operating conditions and on an industrial scale, using two types of charcoal: Reference charcoal and charcoal produced by continuous carbonization (Carboval). The main operating parameters were monitored. Carboval charcoal showed better results for chemical and grain size analysis, better permeability in the blast furnace and lower raw consumption, showing its technical viability.

Keywords: Charcoal; Continuous carbonization; Blast furnace; Performance.

1 Bacharel e Mestre em Estatística, Doutora em Engenharia Metalúrgica, Coordenadora Técnica, Superintendência de Siderurgia, Vallourec Tubos do Brasil, Belo Horizonte, Minas Gerais, Brasil.

2 Graduado e Mestre em Engenharia Metalúrgica, Superintendente, Superintendência de Siderurgia, Vallourec Tubos do Brasil, Belo Horizonte, Minas Gerais, Brasil

3 Graduado, Mestre e Doutor em Engenharia Metalúrgica, Especialista em Engenharia Econômica, Diretor Presidente, RSConsultants Ltda., Belo Horizonte, Minas Gerais, Brasil.

4 Graduado e Mestre em Engenharia Metalúrgica, Dr. Ing., Chief Inovation Officer, Vallourec, Paris, França.

5 Engenheiro Metalúrgico, Gerente, Gerência do Projeto de Carbonização Contínua, Vallourec Florestal, Paraopeba, Minas Gerais, Brasil.
\end{abstract}




\section{INTRODUÇÃO}

O carvão vegetal, CV, como redutor, tem extrema importância na operação de um alto-forno, pois afeta a estabilidade de marcha, a permeabilidade do leito da carga, a produtividade e o consumo total de combustíveis. Além disso, é o componente individual de maior impacto no custo final de produção de ferro-gusa nas usinas integradas.

Neste contexto, foi desenvolvido um processo de carbonização contínua, com uma tecnologia moderna e um conceito próprio de carbonização que a diferencia das carbonizações tradicionais (fornos circulares e retangulares), permitindo que o CV produzido tenha melhores características físico-químicas. Sendo assim, a próxima etapa é a reprodução da planta continua em módulos permitindo uma maior escala de produção de CV com custo competitivo.

Com base nisso, desenvolveu-se este trabalho com o objetivo de avaliar, além da qualidade do CV em laboratório, seu desempenho em um alto-forno em condições reais de operação e em escala industrial. Ou seja, verificar se haveria dificuldade de performance no alto-forno, e não uma comparação termoquímica, mantendo a relação peso do CV enfornado pelo peso da carga metálica constante.

O teste foi feito através da utilização de dois tipos de carvões (CVs) no alto-forno: i) CV produzido em uma fazenda referência (CV Referência) e ii) CV produzido na carbonização contínua da Vallourec, chamado Carboval (SAMPAIO, 2008), buscando avaliar os impactos operacionais e de consumo de CV.

\section{MATERIAIS E MÉTODOS}

Considerando a capacidade do processo de carbonização contínua da Carboval de 20t/dia, foram produzidos $1.725 \mathrm{t}$ de CV durante aproximadamente 86 dias, para serem consumidos em 10 dias consecutivos por um alto-forno.

Para se ter uma base de comparação ao CV Carboval, antes do seu uso, o altoforno operou também durante 10 dias utilizando um CV denominado Referência.

A Figura 1 a seguir mostra os passos da preparação para o teste. 
J
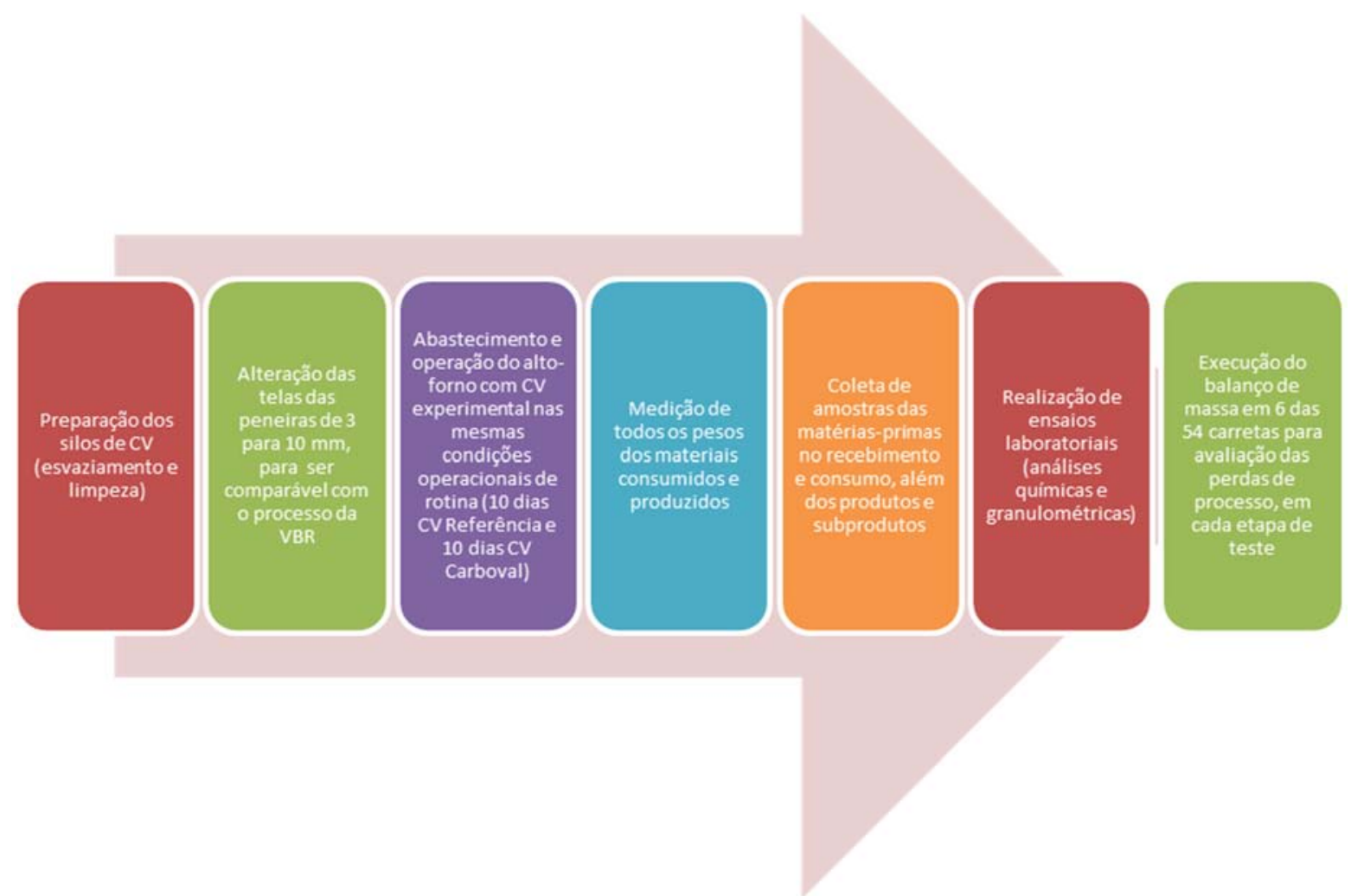

Figura 1. Fluxograma de preparação dos testes.

A Figura 2 ilustra como foi executado o balanço de massa para avaliação das perdas de processo em cada etapa de teste:

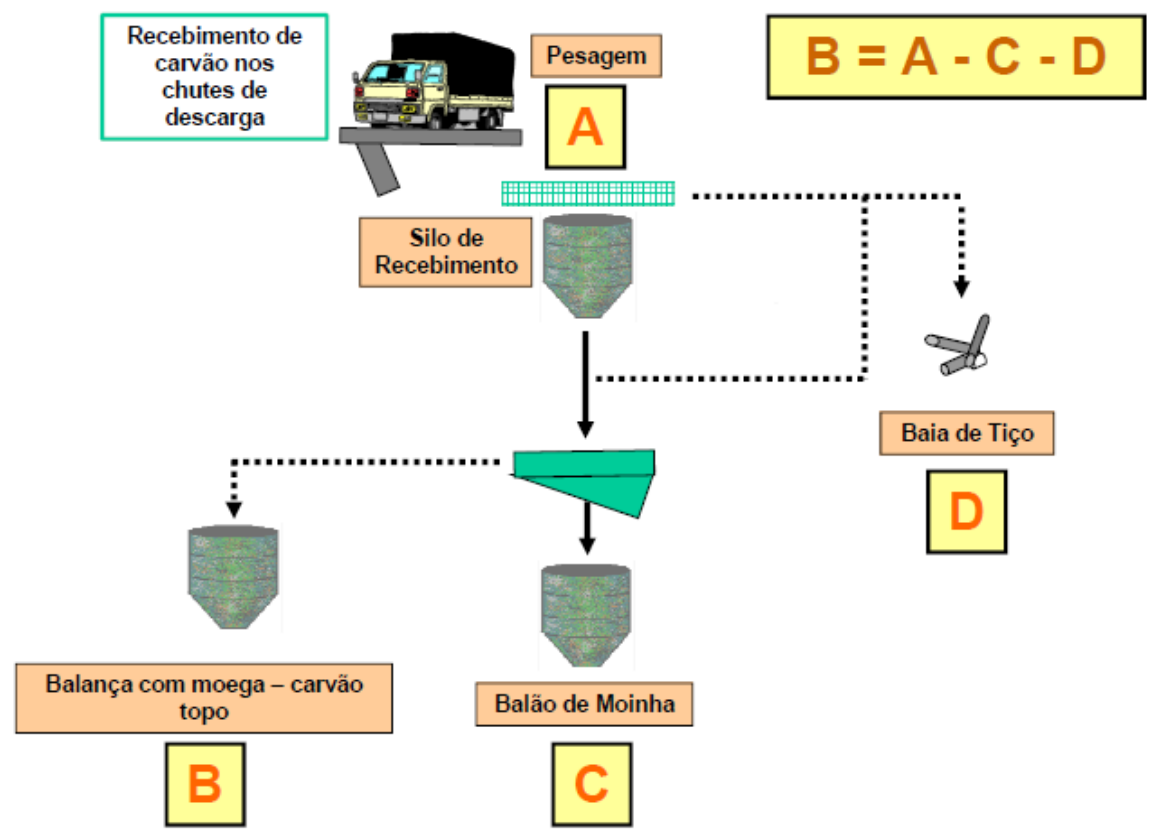

Figura 2. Procedimento do balanço de massas.

O software estatístico MINITAB foi utilizado para a elaboração dos gráficos e a realização dos testes estatísticos. Em termos de comparação de médias, foi realizado o teste t. Para comparar as variâncias, o teste Levene (TRIOLA, 1999). O nível de significância considerado foi de $5 \%$.

Segue Tabela $1 \mathrm{com}$ as principais características do alto-forno: 
Tabela 1. Características do alto-forno utilizado no teste

\begin{tabular}{|c|c|}
\hline Item & Característica \\
\hline Volume útil do alto-forno & $90 \mathrm{~m}^{3}$ \\
\hline Diâmetro do cadinho & $2,7 \mathrm{~m}$ \\
\hline Número de ventaneiras & 6 \\
\hline Diâmetro das ventaneiras & $105 \mathrm{~mm}$ \\
\hline Produção média diária & $240 \mathrm{t}$ \\
\hline Índice de produtividade & 2,67 \\
\hline $\begin{array}{l}\text { Sistema de descarga e abastecimento de } \\
\text { CV }\end{array}$ & $\begin{array}{c}\text { Possui: } \\
2 \text { chutes de descarga de CV } \\
8 \text { silos de } \mathrm{CV} \text {, sendo } 8 \text { bocas de descarga/silo. } \\
\text { No momento da descarga, o CV é peneirado em } \\
\text { tela fixa de } 12 \mathrm{~mm} \text { e depois redirecionado para o } \\
\text { silo de estocagem. }\end{array}$ \\
\hline Sopradores & $\begin{array}{c}5 \text { sopradores de } 150 \mathrm{KW} \text {, sendo } 4 \text { em operação e } \\
1 \text { na reserva. } \\
\text { Opera no limite máximo da vazão de sopro, } \\
\text { mantendo a válvula da linha de ar frio totalmente } \\
\text { aberta. }\end{array}$ \\
\hline Carga metálica para os testes & $\begin{array}{c}\text { Safm }=21,2 \% \\
\text { Polaris }=21,2 \% \\
\text { Minerita }=21,2 \% \\
\text { Briquete de lama de aciaria = } 25 \% \\
\text { Sucata de gusa }=11,4 \%\end{array}$ \\
\hline Faixa de temperatura de gusa objetivada & 1350 a $1380^{\circ} \mathrm{C}$ \\
\hline Sistema de granulação de escória & Sim \\
\hline $\begin{array}{l}\text { Enriquecimento do ar de sopro com } \\
\text { oxigênio }\end{array}$ & Não \\
\hline $\begin{array}{l}\text { Injeção de combustível auxiliar nas } \\
\text { ventaneiras }\end{array}$ & Não \\
\hline Tipo de gusa produzido & Aciaria \\
\hline
\end{tabular}

Foram monitorados os seguintes parâmetros operacionais, objetos de análise deste trabalho:

Carga/hora
Vazão de sopro
Pressão de sopro
Temperatura de sopro
Temperatura de topo
Temperatura de secagem de minério
Densidade do cv enfornado
Umidade do cv enfornado
Tempo de forno aberto
Temperatura do gusa
Permeabilidade
Consumo específico de ar
Produção e composição do gusa

Carga/hora

Vazão de sopro

Temperatura de sopro

Temperatura de topo

Temperatura de secagem de minério

Densidade do cv enfornado

Umidade do cv enfornado

Tempo de forno aberto

Temperatura do gusa

Permeabilidade

Produção e composição do gusa
Produção e composição da escória

Consumo de CV

Qualidade do CV

Geração de pó coletor

Composição, consumo e qualidade da

carga metálica

Consumo de fundente (calcário)

Balanço de CV (perdas, moinha e CV de topo)

Cortes de gaiola e/ou arriamentos

Abertura da válvula de sopro

Distribuição de carga 


\section{RESULTADOS E DISCUSSÃO}

\subsection{Análises imediata e granulométrica}

$\mathrm{Na}$ Figura 3 podem-se ver os resultados de análise imediata e granulométrica referentes ao ano 2015, do CV recebido na Vallourec Tubos do Brasil (VBR).

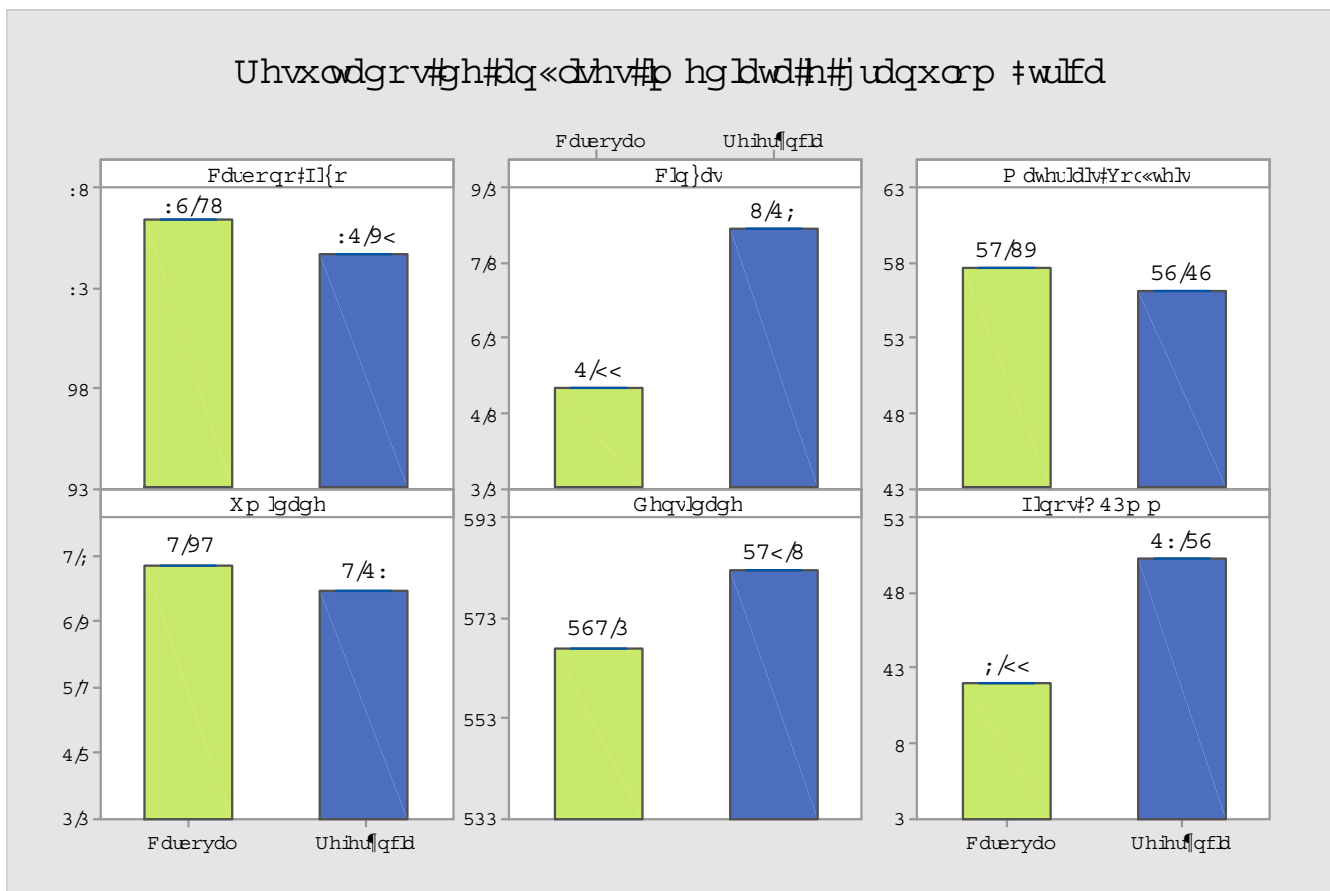

Figura 3. Resultados das análises imediata e granulométrica.

As diferenças em termos de médias foram significativas para carbono fixo (valor- $p=$ 0,007 ), cinzas (valor-p $=0,000$ ), materiais voláteis (valor- $p=0,000$ ), densidade (valor- $p=0,000$ ) e fração de finos $<10 \mathrm{~mm}$ (valor- $p=0,000$ ). Para umidade (valor-p $=0,117$ ) e tamanho médio (valor-p $=0,166$ ), as diferenças entre as médias não foram significativas. Em termos de variabilidade, não houve diferenças significativas entre os resultados.

É importante relatar que o CV Carboval precisou ser colocado no solo para acumular o montante necessário ao experimento. Com isto, o teor de cinzas saiu do valor máximo esperado de $1,0 \%$ para $1,99 \%$, devido à contaminação pelo manuseio e estocagem em solo terroso.

\subsection{Produção}

As produções médias diárias utilizando CV Referência e CV Carboval foram semelhantes em termos de média (valor-p $=0,446$ ) e variabilidade (valor-p $=0,259$ ). A Figura 4 apresenta o gráfico de intervalo de confiança das médias das produções para cada tipo de CV. 


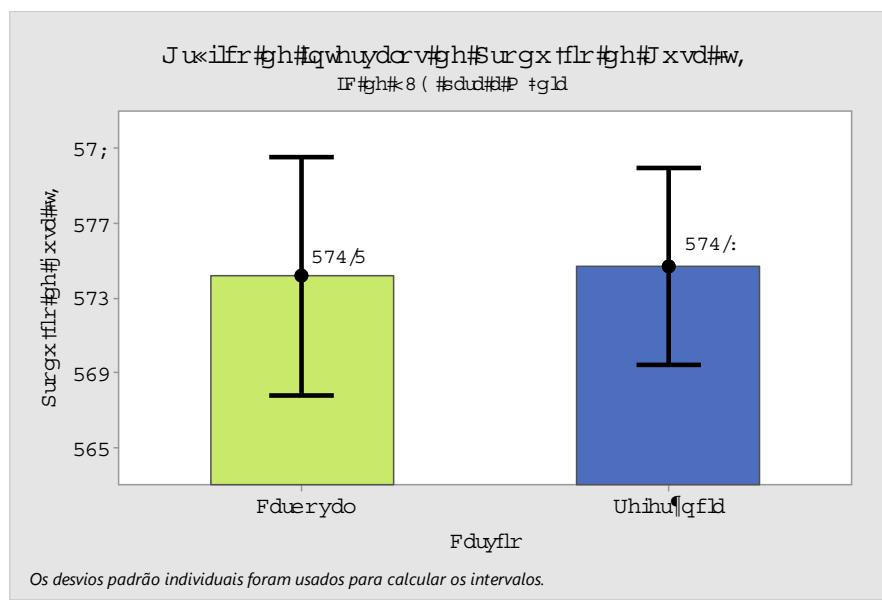

Figura 4. Produção diária de gusa em cada fase do teste.

Com relação à permeabilidade no alto-forno, o CV Carboval apresentou maior média (valor-p $=0,000)$, como pode ser visto na Figura 5 . Em termos de variabilidade não houve diferença (valor- $p=0,256$ ).

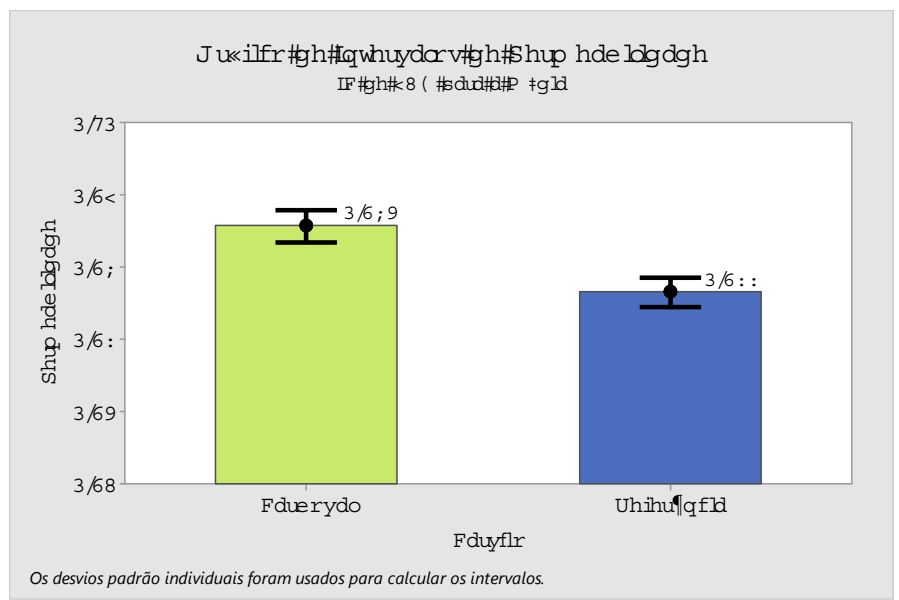

Figura 5. Cálculo da permeabilidade $($ Vsopro/ $\Delta \mathrm{P})$ em cada fase do teste.

Comparando os parâmetros operacionais relacionados com a produção de gusa (Figura 6), o CV Carboval apresentou maior média de vazão de sopro (valor-p = 0,05 ) e de temperatura de topo (valor-p $=0,005$ ). Para os parâmetros carga/hora e ocorrências de engaiolamentos/arriamentos não houve diferença.

Como foi objetivado manter a válvula da rede de ar frio 100\% aberta durante a realização dos testes, assegura-se que a variação na vazão é reflexo da permeabilidade. A pressão de sopro manteve-se constante em torno de $6,7 \mathrm{mCA}$ durante a utilização dos testes com os dois tipos de CV. 


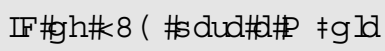

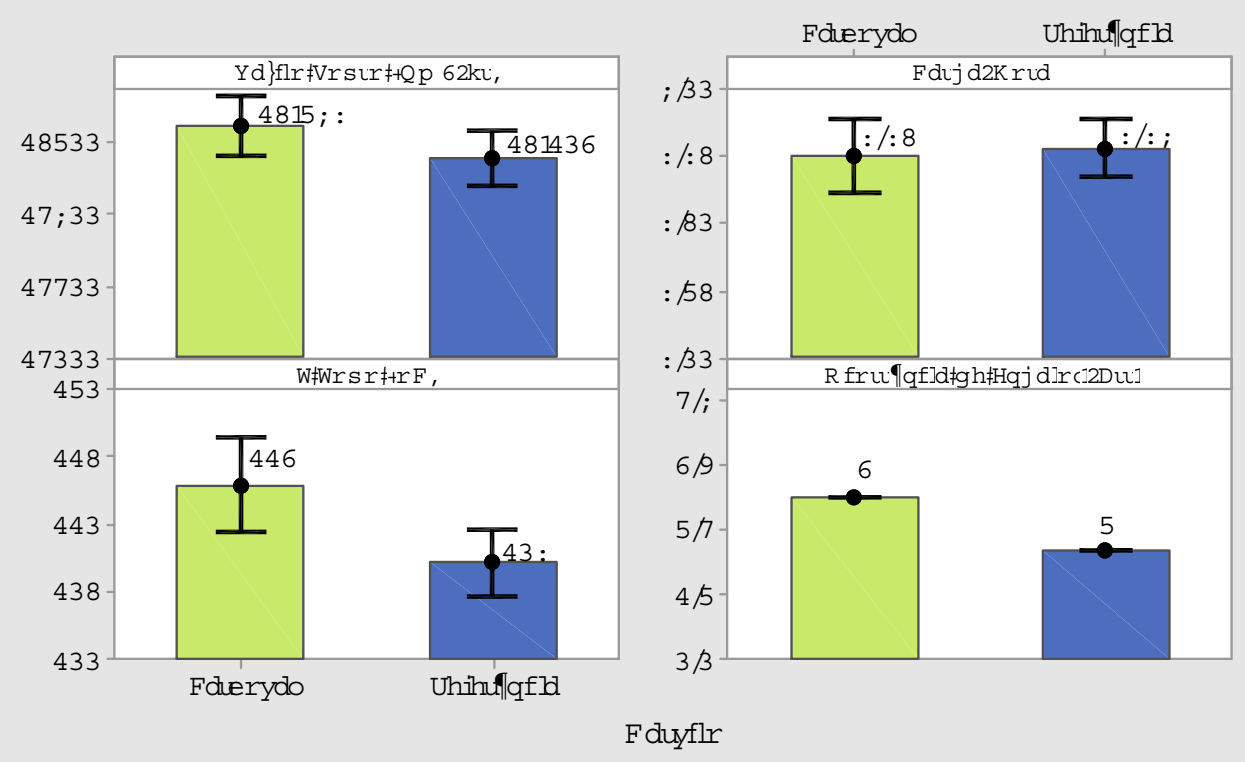

Figura 6. Parâmetros de operação relacionados à produção diária de gusa.

\subsection{Consumo de CV}

Segue abaixo na Figura 7 o consumo de CV bruto do alto-forno por tipo de CV utilizado:

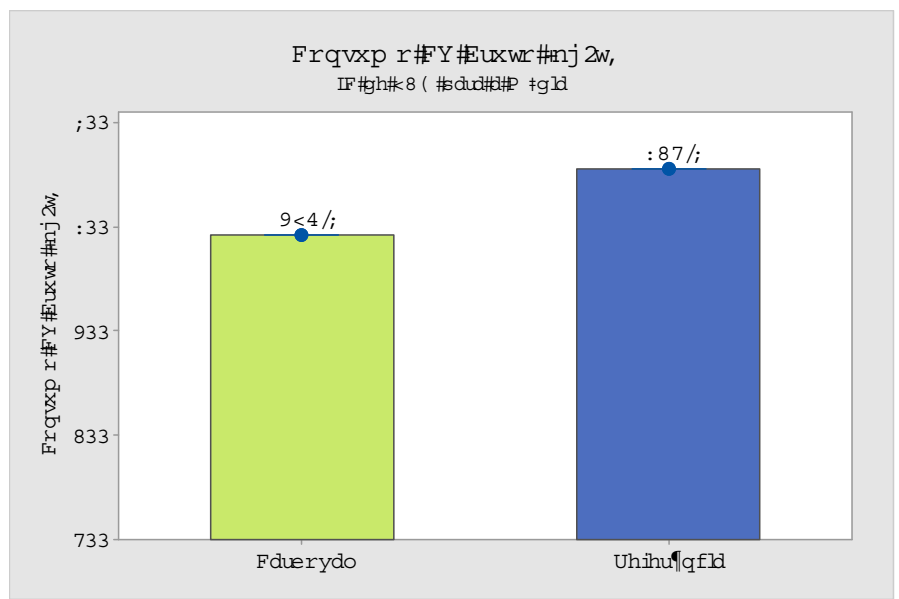

Figura 7. Consumo de CV bruto por tipo de CV.

Verifica-se que o CV Carboval teve menor consumo de CV bruto (diferença de 63 $\mathrm{kg} / \mathrm{t}$ ). Fato justificado pela melhor qualidade química e menor geração de finos. $\mathrm{O}$ total de geração de moinha obtido com a utilização do CV Carboval foi de 144,8 kg/t, enquanto para o CV Referência foi de $235,9 \mathrm{~kg} / \mathrm{t}$.

Durante os testes, dois importantes parâmetros que foram monitorados, temperatura do gusa e temperatura dos gases no topo, apresentaram diferenças significativas durante a operação do alto-forno com os CVs Referência e Carboval (valores-p = $0,000$ e 0,005 , respectivamente). Já as temperaturas de sopro (valor-p $=0,248)$ e de chama (valor-p $=0,248$ ) se mantiveram estáveis (Figura 8). 


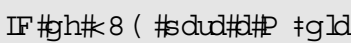

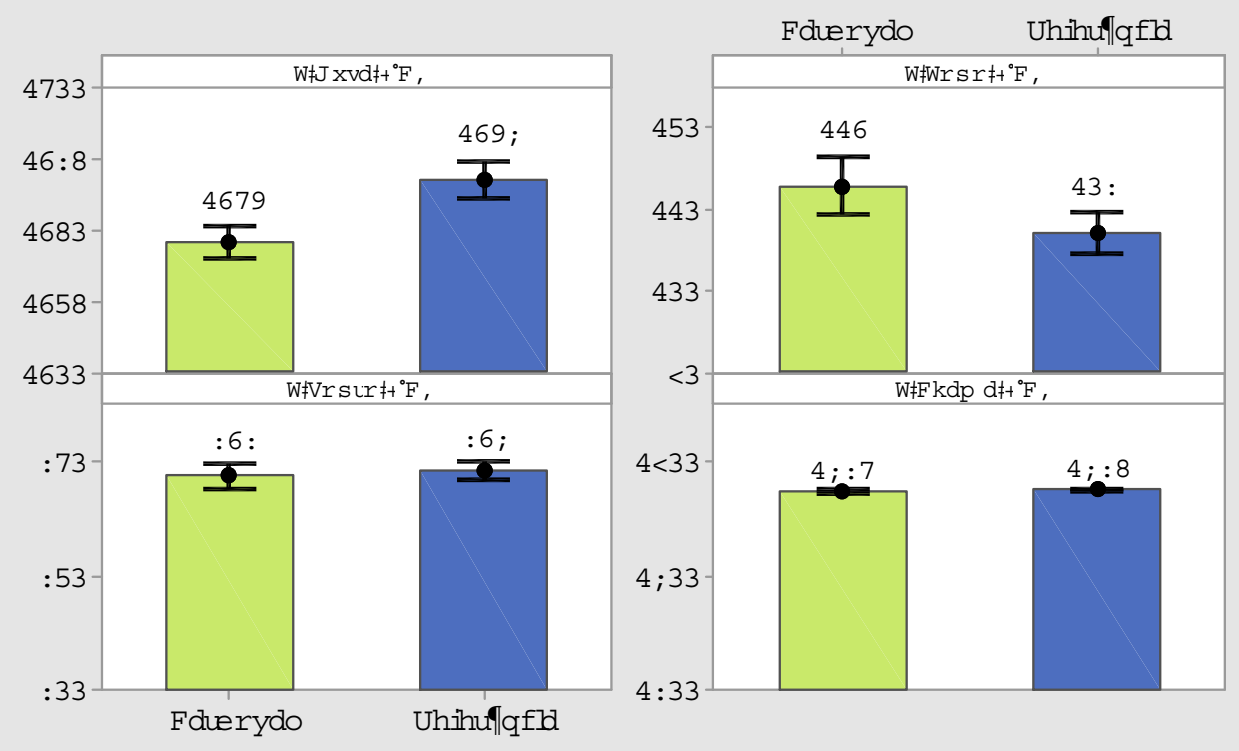

Figura 8. Análise dos parâmetros que afetam o consumo de CV.

A operação com o CV Referência foi conduzida com média de temperatura de gusa mais elevada do que no teste com CV Carboval $\left(1368^{\circ} \mathrm{C}\right.$ e $1346^{\circ} \mathrm{C}$, respectivamente). Isto significa que uma quantidade adicional de energia foi consumida para esse aumento de temperatura, o que poderia ser convertido em consumo de CV.

Por outro lado, a operação com o CV Carboval apresentou média de temperatura de gusa abaixo da faixa de trabalho $\left(1350\right.$ a $\left.1380^{\circ} \mathrm{C}\right)$, o que neste caso gerou economia de $\mathrm{CV}$ em relação ao outro $\mathrm{CV}$ testado.

Temperaturas dos gases no topo mais elevadas significam perda de energia, que poderia ser convertida em redução do consumo de CV. A operação utilizando CV Carboval apresentou média de temperatura de topo mais elevada do que com o CV Referência $\left(113^{\circ} \mathrm{C}\right.$ e $107^{\circ} \mathrm{C}$, respectivamente).

\subsection{Geração de escória e pó coletor}

Não houve diferença significativa entre as gerações de escória e pó coletor dos CVs testados, sendo em média $167 \mathrm{~kg} / \mathrm{t}$ de escória e $63 \mathrm{~kg} / \mathrm{t}$ de pó coletor.

Também não houve alteração significativa na composição da escória ( $\mathrm{SiO}_{2}, \mathrm{Al}_{2} \mathrm{O} 3$, $\mathrm{CaO}, \mathrm{MgO}, \mathrm{FeO}, \mathrm{MnO}$, basicidades binária e terciária), já que o mesmo mix de carga metálica foi utilizado: $21,2 \%$ de cada um dos três minérios, $25 \%$ de briquete de lama e $11,4 \%$ de sucata metálica.

\section{CONCLUSÃO}

Após a realização dos testes, nos quais operou-se um alto-forno por 10 dias utilizando um CV Referência (fornos retangulares), e depois mais 10 dias usando CV Carboval (carbonização contínua), verificou-se que o CV Carboval apresentou melhor desempenho, traduzido por menor consumo bruto (economia de $8,3 \%$ de CV) em função de melhor qualidade química e física. 
O CV Carboval apresentou melhores resultados para as análises imediata e granulométrica, sendo os principais: menor teor de cinzas (consequentemente maior teor de carbono fixo) e menor percentual de finos $<10 \mathrm{~mm}$.

Não houve diferença entre as produções médias diárias de ferro-gusa e gerações de pó coletor e escória utilizando os dois CVs testados

O CV Carboval proporcionou maior permeabilidade no alto-forno, pois permitiu maior vazão de sopro, sem alteração na pressão de sopro.

Conclui-se, portanto, que o CV Carboval foi aprovado em teste industrial demonstrando sua viabilidade técnica.

\section{REFERÊNCIAS}

1 SAMPAIO, R. S. Estudo Prospectivo do Setor Siderúrgico. NT Conversão da Biomassa em Carvão Vegetal Situação Atual. CGEE: Ciência, Tecnologia e Inovação. 2008, Belo Horizonte.

2 TRIOLA, M. F. Introdução à Estatística. Sétima Edição. Livros Técnicos e Científicos. 1999, Rio de Janeiro. 